\title{
Screening wild and cultivated cucurbits against root knot nematode to exploit as rootstocks for grafting in cucumber
}

\author{
C. Thangamani*1 ${ }^{*}$ L. Pugalendhi ${ }^{2}$ and V. Punithaveni ${ }^{3}$ \\ ${ }^{1}$ Horticultural Research Station, Kodaikanal, TNAU, India \\ ${ }^{2}$ Tapioca and Castor Research Station, Yethapur, TNAU, India \\ ${ }^{3}$ Department of Vegetable Crops, Horticultural College \& Research Institute, TNAU, Coimbatore, India. \\ *E-mail: thangamani.sk@gmail.com
}

\begin{abstract}
Yield of mono-cropped cucumber (Cucumis sativus L.) is reduced by root knot nematode (Meloidogyne incognita Kofoid and White). Use of resistant rootstocks in grafting may overcome the problem. Cucurbitaceous species were screened against root knot nematode to evaluate their use as rootstocks in grafting. Inoculation was with nematodes @ $2 \mathrm{~J}_{2} \cdot \mathrm{g}^{-1}\left(\mathrm{~J}_{2}=\right.$ second stage juvenile) of soil in pot culture at the 1 to 2 true leaf stage, 45 days after inoculation, plants were uprooted and observations made to calculate Root Knot Nematode Index (RKI). Cucurbita moschata, Cucumis metuliferus, Citrullus colocynthis and Cucumis callosus were resistant having a RKI-2. Cucurbita ficifolia, Cucurbita maxima, Cucumis melo sub sp. agrestis were moderately resistant with a RKI-3. Total phenols content in roots indicates plant resistance to M. incognita. Cucumis metuliferus had the highest mean total phenols content $(16.98 \mathrm{mg} \cdot \mathrm{g}$ ${ }^{1}$ of root) followed by Citrullus colocynthis $\left(16.08 \mathrm{mg} \cdot \mathrm{g}^{-1}\right.$ of root) and Cucurbita moschata $\left(15.37 \mathrm{mg} \mathrm{g}^{-1}\right.$ of root). Resistant rootstocks possessed higher peroxidase and PPO activity than susceptible ones. Cucumis metuliferus had the highest value of peroxidase and PPO activity $\left(3.83 \mathrm{OD} \cdot \mathrm{min}^{-1} \cdot \mathrm{g}^{-1}\right.$ of root and $3.67 \mathrm{OD} \cdot \mathrm{min}^{-1} \cdot \mathrm{g}^{-1} \mathrm{of}$ root) followed by Citrullus colocynthis $(3.26$ and $3.63 \mathrm{OD} \cdot \mathrm{min}^{-1} \cdot \mathrm{g}^{-1}$ of root), Cucumis callosus $\left(3.02\right.$ and $2.98 \mathrm{OD} \cdot \mathrm{min}^{-1} \cdot \mathrm{g}^{-1}$ of root) and Cucurbita moschata (2.93 and $2.94 \mathrm{OD} \cdot \mathrm{min}^{-1} \cdot \mathrm{g}^{-1}$ of root). Cucumber scions, 'Green Long' and 'NS 408' had lower peroxidase and PPO activity of 0.64 and $1.42 \mathrm{OD} \cdot \mathrm{min}^{-1} \cdot \mathrm{g}^{-1}$ and 0.57 and $1.31 \mathrm{OD} \cdot \mathrm{min}$ ${ }^{1} \cdot \mathrm{g}^{-1}$ of root, respectively. Resistant and moderately resistant cucurbitaceous species may be used for further studies possibly leading to improved yield.
\end{abstract}

Keywords: Meloidogyne incognita - Root Knot Nematode Index - Phenols - Peroxidase - PPO

\section{INTRODUCTION}

Cultivation of cucumber (Cucumis sativus L.) is impaired and yield reductions occur due to the Root Knot Nematode (Meloidogyne incognita Kofoid and White). The nematode damages plants by direct feeding from induced large galls throughout the root system of infected plants and alters uptake of water and nutrients, interfering with translocation of photosynthates. The nematode spends most of their active life within plant roots. The infestation stage is the second-stage juvenile $\left(\mathrm{J}_{2}\right)$, which penetrates the root and migrates to the vascular tissue to establish a permanent feeding site (Williamson and Hussey, 1996). Incidence of $M$. incognita can result in a $25 \%$ annual yield loss in field grown cucumber (Anwar and McKenry, 2012). Control of root-knot nematodes in cucumber with soil fumigants or other nematicides, is becoming more difficult, because of increased cost of chemicals and high toxicity of the chemicals to nearly all living organisms. The degree of resistance due to these chemicals is not acceptable. Alternative approaches for managing this problem in cucumber are necessary.

Grafting is a technique using resistant root stocks to mitigate root knot nematode incidence. Wild Cucumis species and other cucurbitaceous root stocks provide a broad base for grafting. Initial screening studies of root stocks for root knot nematode resistance or tolerance could help in root stock selection. Inducible defense against nematodes includes accumulation of peroxidase (Ibrahim, 1991) and polyphenol oxidase 
(Zacheo and Zacheo, 1995). Biochemical responses during invasion supports this mechanism which occurs due to non-cooperative action of host tissue or cells. Chemical inhibitors in host tissue counteract, or neutralize giant cell inducing effects of nematode salivary secretions (Barons, 1939). This study was undertaken to evaluate root stocks for their resistance to root knot nematode.

\section{MATERIALAND METHODS}

Wild and cultivated cucurbitaceous species rootstocks viz., Cucurbita ficifolia (Fig Leaf Gourd), Cucurbita moschata (Pumpkin), Cucurbita maxima (Winter squash), Cucumis metuliferus (African horned cucumber), Citrullus colocynthis (Colocynth), Cucurbita pepo (Summer squash), Luffa cylindrica (Sponge gourd), Lagenaria siceraria (Bottle gourd), Cucumis callosus, Cucumis melo sub sp. agrestis, Cucumis melo var. momordica (Snap melon), Cucumis melo var. utilissimus (Long melon) and the cucumber scions 'NS 408' and 'Green Long' were screened for resistance to root knot nematode in pot culture. The experiments were carried out during 201314 at the Nematology Glass House, College Orchard, Tamil Nadu Agriculture University (TNAU), Coimbatore in a randomized block design.

Samples of root bearing galls, with their rhizospheric soils, were collected from experimental plots of the Department of Nematology, TNAU. Isolation of $M$. incognita was with Cobb's sieving and decanting method followed by a modified Baermann funnel technique (Cobb, 1918; Schindler, 1961). The identity of $M$. incognita was confirmed taxonomically. Highly susceptible tomato (Solanum lycopersicum L) and cucumber plants were used for developing pure cultures of $M$. incognita. The plants were maintained in pots filled with a steam sterilized loamy soil mixed with fine river sand. Plants were inoculated with 2-3 $\mathrm{J}_{2}$ stage $\mathrm{g}^{-1}$ soil of $M$. incognita race 3 per pot. Rootstocks and scions were initially grown in protrays and 15-day old healthy seedlings transplanted in to plastic pots with $13.73 \mathrm{~cm}$ bottom dia, $17.5 \mathrm{~cm}$ height and $20 \mathrm{~cm}$ top dia containing $5.0 \mathrm{~kg}$ of sterilized pot mixture of red soil:sand:cow manure, 2:2:1 ratio. There were 3 replications for each cultivar. Initially $300 \mathrm{~mL}$ of water used to irrigate pots on alternate days. After establishment of seedlings irrigation was at 3 to 4 day intervals. No chemical fertilizers were applied. To control foliage pests, neem oil @ 3\% was applied twice at biweekly intervals. The method suggested by Sasser et al. (1957) was used to inoculate plants with nematodes. Infested roots from pure culture were cut into pieces about $2 \mathrm{~cm}$ long and placed in $0.5 \%$ sodium hypochlorite $(\mathrm{NaOCl})$ solution. The container was shaken for about $3 \mathrm{~min}$ to dissolve the gelatinous matrix freeing eggs from the egg mass and incubated for 48 $\mathrm{hr}$ under room temperature. The eggs were kept in Petri dishes and an aerator used to enable hatching. The inoculum concentration was adjusted to a known number by addition of water, by taking a known quantity $(1 \mathrm{~mL})$ after shaking and the population counted under a stereoscopic microscope. The nematode inoculum (2 $\mathrm{J}_{2} \mathrm{~g}^{-1}$ of soil) was placed at a $2 \mathrm{~cm}$ depth in the rhizosphere region of soil and covered with sterile sand at 15 days after planting. Plants were uprooted at 45 days after inoculation and washed in a gentle stream of water. Shoot and root length, fresh and dry weights, and root dry weights measured. Dry weight was determined after drying tissues in a forced air oven at $60^{\circ} \mathrm{C}$ for $72 \mathrm{hrs}$. From fresh root samples, numbers of galls $/ 10 \mathrm{~g}$ of root, egg masses and females $\mathrm{g}^{-1}$ of root were counted under a stereoscopic microscope after staining with acid fuchsin lactophenol.

Degree of resistance was assessed as indicated by the root knot index as per the method developed by Heald et al. (1989). Total phenols, peroxidase and polyphenol oxidase were estimated in plants. Recently matured physically active roots of 5 randomly selected plants after inoculation were used for analysis. Root samples obtained at $0,24,48,72,96,120 \mathrm{hrs}$ were homogenized in a chilled pestle and mortar for enzyme extraction.

The Folin-Ciocalteau reagent method was used to estimate total phenols (Bray and Thrope, 1954). One-mL of ethanol root extract was placed in a boiling tube to which $1 \mathrm{~mL}$ of Folin Ciocalteau reagent and 2 $\mathrm{mL}$ of $20 \%$ sodium carbonate were added. The mixture was heated for exactly $1 \mathrm{~min}$ in a boiling water bath at $70^{\circ} \mathrm{C}$. After cooling, $2 \mathrm{~mL}$ of distilled water was added and the blue color development was read at $660 \mathrm{~nm}$ in a UV spectrophotometer.

Peroxidase activity was assayed using the method of Srivastava (1987). The reaction mix consisted of $1.5 \mathrm{~mL}$ guiacol solution, $100 \mu \mathrm{mL}$ peroxidase enzyme preparations and $100 \mu \mathrm{L}$ hydrogen peroxidase $\left(\mathrm{H}_{2} \mathrm{O}_{2}\right)$ was prepared for assay. At the start of enzyme reaction, absorbance of the mixture was 
Table 1. Reaction of wild and cultivated cucurbitaceous rootstocks and cucumber scions to M. incognita on growth parameters at 45 days after inoculation

\begin{tabular}{|c|c|c|c|c|c|c|}
\hline $\begin{array}{l}\text { Cucurbitaceous } \\
\text { species }\end{array}$ & $\begin{array}{l}\text { Mean shoot } \\
\text { length } \\
(\mathrm{cm})\end{array}$ & $\begin{array}{l}\text { Mean shoot } \\
\text { fresh weight } \\
\quad(\mathrm{g})\end{array}$ & $\begin{array}{l}\text { Mean shoot } \\
\text { dry weight } \\
\text { (g) }\end{array}$ & $\begin{array}{l}\text { Mean root } \\
\text { length } \\
\text { (cm) }\end{array}$ & $\begin{array}{l}\text { Mean root } \\
\text { fresh weight } \\
\text { (g) }\end{array}$ & $\begin{array}{c}\text { Mean root } \\
\text { dry weight } \\
\text { (g) }\end{array}$ \\
\hline \multicolumn{7}{|l|}{ Rootstocks } \\
\hline $\begin{array}{l}\text { Fig leaf gourd } \\
\text { (Cucurbita ficifolia) }\end{array}$ & 248.97 & 49.85 & 4.72 & 21.88 & 1.63 & 0.23 \\
\hline $\begin{array}{l}\text { Pumpkin } \\
\text { (Cucurbita moschata) }\end{array}$ & 82.65 & 42.10 & 4.46 & 56.82 & 3.14 & 0.50 \\
\hline $\begin{array}{l}\text { Winter squash } \\
\text { (Cucurbita maxima) }\end{array}$ & 64.53 & 44.91 & 5.03 & 50.95 & 3.05 & 0.36 \\
\hline $\begin{array}{l}\text { African horned cucumber } \\
\text { (Cucumis metuliferus) }\end{array}$ & 148.59 & 19.92 & 2.23 & 16.22 & 0.13 & 0.01 \\
\hline $\begin{array}{l}\text { Colocynth } \\
\text { (Citrullus colocynthis) }\end{array}$ & 74.31 & 8.47 & 0.95 & 22.42 & 1.32 & 0.22 \\
\hline $\begin{array}{l}\text { Summer squash } \\
\text { (Cucurbita pepo) }\end{array}$ & 39.92 & 7.54 & 0.82 & 2.98 & 0.76 & 0.09 \\
\hline $\begin{array}{l}\text { Sponge gourd } \\
\text { (Luffa cylindrica) }\end{array}$ & 88.64 & 19.88 & 2.23 & 39.73 & 1.13 & 0.18 \\
\hline $\begin{array}{l}\text { Bottle gourd } \\
\text { (Lagenaria siceraria) }\end{array}$ & 82.98 & 31.78 & 3.56 & 33.41 & 2.03 & 0.31 \\
\hline Cucumis callosus & 92.94 & 20.81 & 2.12 & 12.93 & 1.71 & 0.53 \\
\hline $\begin{array}{l}\text { Cucumis melo } \\
\text { sub sp. agrestis }\end{array}$ & 90.82 & 14.94 & 1.32 & 11.04 & 0.97 & 0.41 \\
\hline $\begin{array}{l}\text { Snap melon Cucumis } \\
\text { melo var. momordica }\end{array}$ & 96.57 & 18.04 & 2.14 & 23.27 & 0.92 & 0.11 \\
\hline $\begin{array}{l}\text { Long melon Cucumis } \\
\text { melo var. utilissimus }\end{array}$ & 84.02 & 15.69 & 1.86 & 20.24 & 0.80 & 0.10 \\
\hline \multicolumn{7}{|l|}{ Scions } \\
\hline $\begin{array}{l}\text { Cucumis sativus } \\
\text { (Hyb.- NS 408) }\end{array}$ & 93.82 & 15.00 & 1.68 & 13.02 & 0.62 & 0.07 \\
\hline $\begin{array}{l}\text { Cucumis sativus } \\
\text { (Var. Green Long) }\end{array}$ & 109.50 & 22.28 & 2.49 & 20.11 & 0.94 & 0.12 \\
\hline Grand mean & 101.70 & 25.04 & 2.67 & 25.98 & 1.48 & 0.26 \\
\hline SEd & 3.23 & 2.19 & 0.45 & 2.30 & 0.16 & 0.09 \\
\hline $\mathrm{CD}(\mathrm{P}=0.05)$ & 6.67 & 4.53 & 0.94 & 4.74 & 0.32 & 0.19 \\
\hline
\end{tabular}

set to zero at $420 \mathrm{~nm}$ and change in absorbance of samples recorded at $30 \mathrm{sec}$ intervals for $3 \mathrm{~min}$. Boiled enzyme was the control.

Polyphenol oxidase activity was assayed by the method of Srivastava (1987). A standard reaction mixture contained $1.5 \mathrm{~mL}$ of $0.1 \mathrm{M}$ phosphate buffer (pH 6.5), $0.5 \mathrm{~mL}$ enzyme preparation and $0.5 \mathrm{~mL}$ of $0.01 \mathrm{M}$ catechol was prepared. At the start of the enzyme reaction, the absorbance was set to zero at $495 \mathrm{~nm}$. The change in sample absorbance was recorded at $30 \mathrm{sec}$ intervals for $3 \mathrm{~min}$. 
The statistical analysis of the observations recorded was performed according to the method suggested by Panse and Sukhatme (1985). The data were analysed in AGRES statistical package (DOSBox-0.74) developed by TNAU, Coimbatore.

\section{RESULTS AND DISCUSSION}

Rootstocks and scions responded differently for challenge inoculation with root knot nematode (Table $1,2)$. The longest shoots $(248.97 \mathrm{~cm})$ and highest shoot fresh and dry weights $(49.85 ; 4.72 \mathrm{~g})$ were in fig leaf gourd. The shortest shoot length $(39.92 \mathrm{~cm})$, and lowest shoot fresh and dry weight $(7.54 \mathrm{~g} ; 0.82 \mathrm{~g})$ were in summer squash. The Cucurbita species viz., pumpkin and winter squash had longer roots $(56.82 \mathrm{~cm} ; 50.95$ $\mathrm{cm})$ and the highest root fresh and dry weights $\{(3.14 \mathrm{~g}$, $0.5 \mathrm{~g}) ;(3.05 \mathrm{~g}, 0.36 \mathrm{~g})\}$ followed by fig leaf gourd $(21.88 \mathrm{~cm}, 1.63 \mathrm{~g}, 0.23 \mathrm{~g})$ respectively. This might be due to low nematode reproduction on pumpkin, winter squash and fig leaf gourd roots. Tamilselvi (2013) also reported that among the nematode inoculated cucurbits, mean root fresh weight were not reduced due to low nematode reproduction on species like colocynth, African horned cucumber and pumpkin roots.

Among the two scions, the lowest root fresh and dry weights were in cucumber scion 'Green Long' $(0.94 \mathrm{~g} ; 0.12 \mathrm{~g})$. In susceptible species the deformed root system is due to infestation by $M$. incognita which causes development of giant cells and block age of xylem vessels. This interferes with nutrient uptake and results in impaired plant growth. These findings agree with Ploeg and Phillips (2001) in melons species and Krishnaveni and Subramanian (2002) in cucumber where more than $1000 \mathrm{~J}_{2}$ of $M$. incognita as initial inoculation caused significant reduction in shoot length, root length and shoot weight.

All cucurbitaceous species rootstocks and cucumber scions developed characteristic galls caused by root knot nematode (Table 2). There were differences among rootstocks for number of galls, egg masses and females $\mathrm{g}^{-1}$ of root. Colocynth had the lowest numbers of galls/10 g of roots (4.69) followed by African horned cucumber (5.24) and the wild Cucumis callosus (8.36) compared to other cucurbitaceous species and cucumber scions. However, lesser galls/10 g of roots was observed fig leaf gourd (25.32), winter squash (11.32), Cucumis melo sub sp. agrestis (29.34). Nematode egg production indicated at nematodes completed their life cycle within the roots. Numbers of egg masses (1.94) and root knot nematode females (3.85) were the lowest on African horned cucumber followed by colocynth $(1.98,4.81)$ and Cucumis callosus $(3.12,5.44)$. Whereas, reduction in numbers of galls, females and egg-masses were observed in winter squash followed by fig leaf gourd by Amin et al. (2012). This may be due to poor host status for the invading parasite (Siguenza et al., 2005). In the susceptible species summer squash, cucumber, bottle gourd, long melon and snap melon had higher numbers of galls, eggs and females compared to moderately resistant and resistant species (Table 2).

The genotypes had variable Root Knot Index (RKI) values. Lower number of gall as observed in African horned cucumber, colocynth, Cucumis callosus and pumpkin classified them as resistant to root knot nematode with a RKI-2. Whereas, lesser galls/10 $\mathrm{g}$ of roots was observed fig leaf gourd, winter squash and Cucumis melo sub sp. agrestis were classified as moderately resistant with a RKI-3. Resistance reaction might be due to the presence of nematode resistant gene (Hadisoeganda and Sasser, 1982; Roberts and May, 1986) making plants less attractive to attack by nematodes. The compatible and incompatible reactions might be due to presence of resistant genes, which were activated as a result of nematode invasion (Williamson, 1999; Davis et al., 2000; Williamson and Kumar, 2006).

Plants generally respond to nematode invasion by activation of a series of local and systemic defense mechanisms (Trudgill, 1995). Phenolic compounds in nematode injured areas accumulate and associated oxidative enzymes are activated (Balasubramanian and Purushothaman, 1972). Significant difference in root total phenol, peroxidase and polyphenol oxidase activity occurred (Table 3,4,5). Total phenol content in roots is an indication of plant resistance to Meloidogyne incognita. The total phenols content in genotypes varied with respect to inoculation of nematodes (Table 3). African horned cucumber had the highest mean total phenols content (16.98 $\mathrm{mg} \cdot \mathrm{g}^{-1}$ of root) followed by colocynth(16.08 $\mathrm{mg} \cdot \mathrm{g}^{-1}$ of root) and pumpkin $\left(15.37 \mathrm{mg} \cdot \mathrm{g}^{-1}\right.$ of root). Total phenols content in roots appeared to have a negative association with root knot index, number of females and number of egg masses. 
Table 2. Reaction of cucurbitaceous rootstocks and cucumber scions on the incidence of root knot nematode (M. incognita) under pot culture and enzyme levels

\begin{tabular}{|c|c|c|c|c|c|}
\hline $\begin{array}{l}\text { Cucurbitaceous } \\
\text { species }\end{array}$ & $\begin{array}{l}\text { No. of } \\
\text { galls } / 10 \mathrm{~g} \\
\text { of roots }\end{array}$ & $\begin{array}{c}\text { No. of egg } \\
\text { masses/g } \\
\text { of roots }\end{array}$ & $\begin{array}{l}\text { No. of } \\
\text { females } \\
5 \mathrm{~g} \text { root }\end{array}$ & $\mathrm{RKI} *$ & Reaction** \\
\hline \multicolumn{6}{|l|}{ Rootstocks } \\
\hline $\begin{array}{l}\text { Fig leaf gourd } \\
\text { (Cucurbita ficifolia) }\end{array}$ & 25.32 & 11.36 & 6.32 & 3 & MR \\
\hline $\begin{array}{l}\text { Pumpkin } \\
\text { (Cucurbita moschata) }\end{array}$ & 9.26 & 3.61 & 5.94 & 2 & $\mathrm{R}$ \\
\hline $\begin{array}{l}\text { Winter squash } \\
\text { (Cucurbita maxima) }\end{array}$ & 11.32 & 3.94 & 6.50 & 3 & MR \\
\hline $\begin{array}{l}\text { African horned cucumber } \\
\text { (Cucumis metuliferus) }\end{array}$ & 5.24 & 1.94 & 3.85 & 2 & $\mathrm{R}$ \\
\hline $\begin{array}{l}\text { Colocynth } \\
\text { (Citrullus colocynthis) }\end{array}$ & 4.69 & 1.98 & 4.81 & 2 & $\mathrm{R}$ \\
\hline $\begin{array}{l}\text { Summer squash } \\
\text { (Cucurbita pepo) }\end{array}$ & 98.35 & 31.32 & 59.21 & 4 & $S$ \\
\hline $\begin{array}{l}\text { Sponge gourd } \\
\text { (Luffacylindrica) }\end{array}$ & 34.39 & 11.14 & 25.63 & 4 & $S$ \\
\hline $\begin{array}{l}\text { Bottle gourd } \\
\text { (Lagenaria siceraria) }\end{array}$ & 79.36 & 26.27 & 51.94 & 4 & $S$ \\
\hline Cucumis callosus & 8.36 & 3.12 & 5.44 & 2 & $\mathrm{R}$ \\
\hline $\begin{array}{l}\text { Cucumis melo sub } \\
\text { sp. agrestis }\end{array}$ & 29.34 & 10.29 & 22.39 & 3 & MR \\
\hline $\begin{array}{l}\text { Snap melon } \\
\text { Cucumis melo var. momordica }\end{array}$ & 41.20 & 12.36 & 37.51 & 4 & $S$ \\
\hline $\begin{array}{l}\text { Long melon } \\
\text { Cucumis melo var. utilissimus }\end{array}$ & 49.65 & 15.92 & 49.32 & 4 & $S$ \\
\hline \multicolumn{6}{|l|}{ Scions } \\
\hline $\begin{array}{l}\text { Cucumis sativus } \\
\text { (Hyb.- NS 408) }\end{array}$ & 83.25 & 29.35 & 59.31 & 4 & $S$ \\
\hline $\begin{array}{l}\text { Cucumis sativus } \\
\text { (Var. Green Long) }\end{array}$ & 76.35 & 23.39 & 48.35 & 4 & $S$ \\
\hline
\end{tabular}

RKI * - Root Knot Nematode Index (Heald et al., 1989) $\quad * *_{-}$- R- Resistant, MR- Moderately resistant, S-Susceptible 
Screening cucumber rootstocks for nematode resistance

Table 3. Root total phenols content in cucurbitaceous rootstocks and cucumber scions under root knot nematode (M.incognita) inoculation

\begin{tabular}{|c|c|c|c|c|c|c|c|}
\hline \multirow{3}{*}{$\begin{array}{l}\text { Rootstocks/Scions } \\
\text { Rootstocks }\end{array}$} & \multicolumn{7}{|c|}{ Total phenol ( $\mathrm{mg} \mathrm{g}^{-1}$ of fresh roots) } \\
\hline & \multicolumn{7}{|c|}{ Hours after inoculation } \\
\hline & 0 & 24 & 48 & 72 & 96 & 120 & Mean \\
\hline $\begin{array}{l}\text { Fig leaf gourd } \\
\text { (Cucurbita ficifolia) }\end{array}$ & 9.26 & 10.13 & 10.87 & 13.87 & 17.11 & 15.86 & 12.85 \\
\hline $\begin{array}{l}\text { Pumpkin11.87 } \\
\text { (Cucurbita moschata) }\end{array}$ & 12.34 & 12.98 & 15.14 & 21.2 & 18.67 & 15.37 & \\
\hline $\begin{array}{l}\text { Winter squash } \\
\text { (Cucurbita maxima) }\end{array}$ & 9.76 & 11.41 & 11.97 & 12.65 & 13.95 & 12.02 & 11.96 \\
\hline $\begin{array}{l}\text { African horned cucumber } \\
\text { (Cucumis metuliferus) }\end{array}$ & 15.15 & 16.82 & 18.26 & 18.09 & 17.13 & 16.43 & 16.98 \\
\hline $\begin{array}{l}\text { Colocynth } \\
\text { (Citrullus colocynthis) }\end{array}$ & 12.34 & 13.56 & 16.12 & 17.97 & 18.89 & 17.6 & 16.08 \\
\hline $\begin{array}{l}\text { Summer squash } \\
\text { (Cucurbita pepo) }\end{array}$ & 5.21 & 5.94 & 6.84 & 7.98 & 8.12 & 7.79 & 6.98 \\
\hline $\begin{array}{l}\text { Sponge gourd } \\
(\text { Luffa cylindrica) }\end{array}$ & 7.13 & 8.26 & 9.84 & 11.81 & 10.82 & 10.7 & 9.76 \\
\hline $\begin{array}{l}\text { Bottle gourd } \\
\text { (Lagenaria siceraria) }\end{array}$ & 4.42 & 4.87 & 5.86 & 7.43 & 6.86 & 5.12 & 5.76 \\
\hline Cucumis callosus & 7.29 & 7.73 & 9.56 & 9.92 & 9.98 & 9.34 & 8.97 \\
\hline $\begin{array}{l}\text { Cucumis melo sub sp. } \\
\text { agrestis }\end{array}$ & 6.46 & 6.93 & 7.56 & 8.64 & 9.76 & 8.35 & 7.95 \\
\hline $\begin{array}{l}\text { Snap melon } \\
\text { (Cucumis melo var. } \\
\text { momordica) }\end{array}$ & 5.17 & 6.33 & 7.25 & 8.73 & 9.43 & 7.79 & 7.45 \\
\hline $\begin{array}{l}\text { Long melon } \\
\text { (Cucumis melo var. } \\
\text { utilissimus) }\end{array}$ & 5.43 & 6.14 & 7.65 & 8.76 & 9.35 & 7.91 & 7.54 \\
\hline \multicolumn{8}{|l|}{ Scions } \\
\hline $\begin{array}{l}\text { Cucumis sativus } \\
\text { (NS 408) }\end{array}$ & 4.53 & 6.29 & 6.7 & 7.36 & 8.95 & 7.27 & 6.85 \\
\hline $\begin{array}{l}\text { Cucumis sativus } \\
\text { (Green Long) }\end{array}$ & 4.87 & 5.63 & 6.85 & 7.96 & 9.03 & 7.78 & 7.02 \\
\hline SEd & 0.19 & 0.49 & 0.32 & 0.50 & 0.53 & 0.48 & \multirow{2}{*}{-} \\
\hline $\mathrm{CD}(P=0.05)$ & 0.39 & 1.01 & 0.66 & 1.03 & 1.10 & 0.98 & \\
\hline
\end{tabular}


Table 4. Root peroxidase content in cucurbitaceous rootstocks and cucumber scions after root knot nematode (M.incognita) inoculation

\begin{tabular}{|c|c|c|c|c|c|c|c|}
\hline \multirow{3}{*}{$\begin{array}{l}\text { Rootstocks/Scions } \\
\text { Rootstocks }\end{array}$} & \multicolumn{7}{|c|}{ Peroxidase (changes in OD. $\mathrm{min}^{-1} \mathrm{~g}^{-1}$ of fresh root) } \\
\hline & \multicolumn{7}{|c|}{ Hours after inoculation } \\
\hline & 0 & 24 & 48 & 72 & 96 & 120 & Mean \\
\hline $\begin{array}{l}\text { Fig leaf gourd } \\
\text { (Cucurbita ficifolia) }\end{array}$ & 1.31 & 1.73 & 2.47 & 2.85 & 2.94 & 2.56 & 2.31 \\
\hline $\begin{array}{l}\text { Pumpkin } \\
\text { (Cucurbita moschata) }\end{array}$ & 1.85 & 2.32 & 2.59 & 3.26 & 3.91 & 3.65 & 2.93 \\
\hline $\begin{array}{l}\text { Winter squash } \\
\text { (Cucurbita maxima) }\end{array}$ & 1.32 & 1.76 & 2.21 & 2.35 & 2.8 & 2.94 & 2.23 \\
\hline $\begin{array}{l}\text { African horned cucumber } \\
\text { (Cucumis metuliferus) }\end{array}$ & 3.15 & 3.29 & 3.56 & 3.92 & 4.34 & 4.72 & 3.83 \\
\hline $\begin{array}{l}\text { Colocynth } \\
\text { (Citrullus colocynthis) }\end{array}$ & 2.42 & 2.87 & 3.09 & 3.18 & 3.74 & 4.26 & 3.26 \\
\hline $\begin{array}{l}\text { Summer squash } \\
\text { (Cucurbita pepo) }\end{array}$ & 1.23 & 1.37 & 1.62 & 2.15 & 2.18 & 2.61 & 1.86 \\
\hline $\begin{array}{l}\text { Sponge gourd } \\
\text { (Luffa cylindrica) }\end{array}$ & 1.12 & 1.38 & 1.65 & 2.32 & 2.54 & 2.69 & 1.95 \\
\hline $\begin{array}{l}\text { Bottle gourd } \\
\text { (Lagenaria siceraria) }\end{array}$ & 1.87 & 2.17 & 2.61 & 2.93 & 3.15 & 2.99 & 2.62 \\
\hline Cucumis callosus & 2.31 & 2.57 & 2.84 & 3.02 & 3.73 & 3.65 & 3.02 \\
\hline Cucumis melo sub sp. agrestis & 1.43 & 1.89 & 2.76 & 2.98 & 3.85 & 3.47 & 2.73 \\
\hline $\begin{array}{l}\text { Snap melon } \\
\text { (Cucumis melo var. } \\
\text { momordica) }\end{array}$ & 1.21 & 1.41 & 2.09 & 2.16 & 2.46 & 2.85 & 2.03 \\
\hline $\begin{array}{l}\text { Long melon } \\
\text { (Cucumis melo var. } \\
\text { utilissimus) }\end{array}$ & 0.89 & 1.24 & 1.76 & 2.15 & 2.45 & 2.08 & 1.76 \\
\hline \multicolumn{8}{|l|}{ Scions } \\
\hline $\begin{array}{l}\text { Cucumis sativus } \\
\text { (NS 408) }\end{array}$ & 0.37 & 0.41 & 0.48 & 0.69 & 0.76 & 0.71 & 0.57 \\
\hline $\begin{array}{l}\text { Cucumis sativus } \\
\text { (Green Long) }\end{array}$ & 0.44 & 0.51 & 0.71 & 0.74 & 0.77 & 0.67 & 0.64 \\
\hline SEd & 0.50 & 0.06 & 0.64 & 0.08 & 0.09 & 0.11 & \multirow{2}{*}{-} \\
\hline $\mathrm{CD}(P=0.05)$ & 1.01 & 0.12 & 1.27 & 0.15 & 0.17 & 0.22 & \\
\hline
\end{tabular}


Screening cucumber rootstocks for nematode resistance

Table 5. Root peroxidase content in cucurbitaceous rootstocks and cucumber scions after root knot nematode (M.incognita) inoculation

\begin{tabular}{|c|c|c|c|c|c|c|c|}
\hline \multirow{3}{*}{$\begin{array}{l}\text { Rootstocks/Scions } \\
\text { Rootstocks }\end{array}$} & \multicolumn{7}{|c|}{ Peroxidase (changes in OD. $\mathrm{min}^{-1} \mathrm{~g}^{-1}$ of fresh root) } \\
\hline & \multicolumn{7}{|c|}{ Hours after inoculation } \\
\hline & 0 & 24 & 48 & 72 & 96 & 120 & Mean \\
\hline $\begin{array}{l}\text { Fig leaf gourd } \\
\text { (Cucurbita ficifolia) }\end{array}$ & 1.69 & 2.43 & 2.74 & 2.87 & 2.94 & 3.05 & 2.62 \\
\hline $\begin{array}{l}\text { Pumpkin } \\
\text { (Cucurbita moschata) }\end{array}$ & 1.85 & 2.32 & 2.59 & 3.26 & 3.91 & 3.65 & 2.93 \\
\hline $\begin{array}{l}\text { Winter squash } \\
\text { (Cucurbita maxima) }\end{array}$ & 1.22 & 1.35 & 1.56 & 1.97 & 2.25 & 2.75 & 1.85 \\
\hline $\begin{array}{l}\text { African horned cucumber } \\
\text { (Cucumis metuliferus) }\end{array}$ & 2.95 & 3.19 & 3.56 & 4.12 & 4.34 & 3.86 & 3.67 \\
\hline $\begin{array}{l}\text { Colocynth } \\
\text { (Citrullus colocynthis) }\end{array}$ & 3.12 & 3.38 & 3.57 & 3.74 & 3.79 & 4.18 & 3.63 \\
\hline $\begin{array}{l}\text { Summer squash } \\
\text { (Cucurbita pepo) }\end{array}$ & 0.35 & 0.45 & 0.62 & 0.72 & 0.92 & 0.84 & 0.65 \\
\hline $\begin{array}{l}\text { Sponge gourd } \\
\text { (Luffa cylindrica) }\end{array}$ & 0.39 & 0.52 & 0.68 & 0.94 & 1.02 & 0.95 & 0.75 \\
\hline $\begin{array}{l}\text { Bottle gourd } \\
\text { (Lagenaria siceraria) }\end{array}$ & 0.41 & 0.59 & 0.75 & 0.98 & 1.23 & 1.62 & 0.93 \\
\hline Cucumis callosus & 2.29 & 2.54 & 2.73 & 3.14 & 3.73 & 3.45 & 2.98 \\
\hline Cucumis melo sub sp. agrestis & 1.41 & 1.78 & 2.65 & 2.87 & 3.72 & 3.41 & 2.64 \\
\hline $\begin{array}{l}\text { Snap melon } \\
\text { (Cucumis melo var. } \\
\text { momordica) }\end{array}$ & 0.64 & 0.98 & 1.11 & 1.89 & 2.16 & 2.46 & 1.54 \\
\hline $\begin{array}{l}\text { Long melon } \\
\text { (Cucumis melo var. } \\
\text { utilissimus) }\end{array}$ & 0.87 & 1.21 & 1.36 & 1.64 & 2.17 & 2.65 & 1.65 \\
\hline \multicolumn{8}{|l|}{ Scions } \\
\hline $\begin{array}{l}\text { Cucumis sativus } \\
\text { (NS 408) }\end{array}$ & 0.67 & 0.85 & 0.93 & 1.18 & 1.88 & 2.35 & 1.31 \\
\hline $\begin{array}{l}\text { Cucumis sativus } \\
\text { (Green Long) }\end{array}$ & 0.73 & 0.82 & 1.26 & 1.57 & 1.73 & 2.41 & 1.42 \\
\hline SEd & 0.39 & 0.08 & 0.70 & 0.12 & 0.19 & 0.09 & \multirow{2}{*}{-} \\
\hline $\mathrm{CD}(P=0.05)$ & 0.80 & 0.16 & 0.14 & 0.24 & 0.38 & 0.18 & \\
\hline
\end{tabular}


Resistant rootstocks possessed higher peroxidase and PPO activity than susceptible ones. African horned cucumber had the highest value of peroxidase and PPO activity $\left(3.83 \mathrm{OD} \cdot \mathrm{min}^{-1} \cdot \mathrm{g}^{-1}\right.$ of root and $3.67 \mathrm{OD} \cdot \mathrm{min}^{-1} \cdot \mathrm{g}^{-1}$ of root) followed by colocynth (3.26 and $3.63 \mathrm{OD} \cdot \mathrm{min}^{-1} \cdot \mathrm{g}^{-1}$ of root), Cucumis callosus (3.02 and $2.98 \mathrm{OD} \cdot \mathrm{min}^{-1} \cdot \mathrm{g}^{-1}$ of root) and pumpkin (2.93 and $2.94 \mathrm{OD} \cdot \mathrm{min}^{-1} \cdot \mathrm{g}^{-1}$ of root). Cucumber scions, 'Green Long' and 'NS 408' had lower peroxidase and PPO activity of 0.64 and 1.42 $\mathrm{OD} \cdot \mathrm{min}^{-1} \cdot \mathrm{g}^{-1}$ of root and 0.57 and $1.31 \mathrm{OD} \cdot \mathrm{min}^{-1} \cdot \mathrm{g}^{-1}$ of root, respectively (Table 4, 5). Several of the genotypes were classified as resistant or moderately resistant. They may be promising candidates to be used as rootstocks for cucumber to enhance resistance to root knot nematodes. Further study on graft compatibility between root knot nematode resistant rootstocks with cucumber is needed.

\section{ACKNOWLEDEMENTS}

The authors acknowledge the financial assistance from The Department of Science and Technology, New Delhi through the Women Scientist Scheme (A) on "Standardization of grafting techniques in cucumber to mitigate root knot nematode and soil borne diseases" and the above research work is part of the scheme.

\section{REFERENCES}

Amin, A.W., A. Wanis, M. Tomader, and G. Abdel Rahman. 2012. Evaluation of some cucurbitaceous rootstocks. 1 - For resistance/ susceptibility to root-knot nematode and fusarium wilt under screenhouse conditions. Egyptian J. Agric. Res. 90(4):1561-1577.

Anwar, S.A. and M.V. McKenry, 2012. Incidence and population density of plant-parasitic nematodes infecting vegetable crops and associated yield losses. Pakistan J. Zool. 44:327-333.

Balasubramanian, M. and D. Purushothaman. 1972. Phenolic contents of root knot affected tissues. Indian J. Nematol. 2:77-94.

Barons, K.C. 1939. Studies of the nature of root knot resistance. J. Agric. Res. 58:263-271.

Bray, H.G. and W.V. Thrope. 1954. Analysis of phenolic compounds of interest in metabolism. Meth. Biochem. Anal. 1:27-52.

Cobb, N.A. 1918. Estimating the nematode population of soil. Technology Circular 1, U.S. Department of Agriculture, Washington, D.C.

Davis, E.L., R.S. Hussey, T.J. Baum, J. Bakker, A. Schots, M.N. Rosso and P. Abad. 2000. Nematode parasitism genes. Ann. Rev. Phytopath. 38:365-396.

Hadisoeganda, W.W. and J.N. Sasser. 1982. Resistance of tomato, bean, southern pea and garden pea cultivars to root knot nematodes based on host suitability. Plant Dis. 66:145150.

Heald, C.M., B.D. Bruton and R.M. Davis. 1989. Influence of Glomus intradices and soil phosphorus on $M$. incognita infecting Cucumis melo. J. Nematol. 21:69-73.

Ibrahim, K.S. 1991. Peroxidase isoenzymes from Meloidogyne cultured on different hosts. Rev. Nematol. 14:335-344.

Krishnaveni, M. and S. Subramanian. 2002. Rootknot nematodes of cucurbits and their management. National symposium on biodiversity and management of nematodes in cropping systems for sustainable agriculture. Department of Nematology, Agricultural Research Station, 11-13 November 2002, Durgapura, Jaipur, India

Panse, V.G. and P.V. Sukhatme. 1957. Statistical methods for agricultural workers. Indian Council of Agricultural Research, New Delhi.

Ploeg, A.T. and M.S. Phillips. 2001. Damage to melon (Cucumis melo L.) cv. Durango by Meloidogyne incognita in Southern California. Nematology 3:151-158.

Roberts, P.A. and D. May. 1986. Meloidogyne incognita resistance characteristics in tomato genotypes developed for processing. $J$. Nematol., 18: 173-178. 
Sasser, J.N., H.R. Powers and G.B. Lucas. 1957. Effect of root knot nematodes on the expression of black shank resistance in tobacco. Physiopathology 43:483-89.

Siguenza, C., M. Schochow, T. Turini, and A. Ploeg.2005. Use of Cucumis metuliferus as a rootstock for melon to manage Meloidogyne incognita. J. Nematol. 37:276 -280 .

Schindler, A.F. 1961. A simple substitute for a Baermann funnel. Plant Dis. Rptr. 45:747748.

Srivastava, S.K. 1987. Peroxidase and polyphenol oxidase in Brassica juncea plants infected with Macrophomina phaseolina (Tassi.) Goid and their implication in disease resistance. J. Phytopath. 120:249-254.
Tamilselvi, N.A.T. 2013. Grafting studies in bitter gourd (Momordica charantia L.). PhD (Horticulture) Thesis, Department of Vegetable Crops, Tamil Nadu Agricultural University, Coimbatore.

Trudgill, D.L. 1995. Origins of root-knot nematodes (Meloidogyne spp.) in relation to their cultural control. Phytoparasitica 23:191-194.

Williamson, V.M. 1999. Plant nematode resistance genes. Curr. Plant Biol. 2:327-331.

Williamson, V.M. and A. Kumar. 2006. Nematode resistance in plants: The battle underground. Trends Genet. 22:396-403.

Williamson, V.M. and R.S. Hussey. 1996. Nematode pathogenesis and resistance in plants. Plant Cell, 8:1735-1745.

Zacheo, G. and T.B. Zacheo. 1995. Plant-nematode interactions: Histological, physiological and biochemical interactions, pp. 321-353, In: K. Kohmoto, U.M., Singh, and R.P. Singh (eds.). Pathogenesis and host specificity in plant diseases. Elsevier Science, Oxford, UK.

(MS Received 16 January 2016, Revised 11 April 2018, Accepted 21 May 2018) 